\title{
Stress-Induced Laboratory Eating Behavior in Obese and Normal-Weight Women
}

\author{
Kießl G and Laessle $\mathrm{R}^{*}$
}

Department of Biological and Clinical Psychology, University of Trier, Trier, Germany

*Corresponding author: Laessle R, Department of Biological and Clinical Psychology, University of Trier, 54286 Trier, Germany, Tel: +49 (0)651/201-2009, E-mail: laessle@uni-trier.de

Citation: Kießl G, Laessle R (2017) Stress-Induced Laboratory Eating Behavior in Obese and Normal-Weight

Women. J Obes Overweig 3(2): 205. doi: 10.15744/2455-7633.3.205

Received Date: November 22, 2017 Accepted Date: December 20, 2017 Published Date: December 22, 2017

\begin{abstract}
Inconsistent results of stress induced eating behavior in obesity have been reported. In order to describe the effect of stress on eating behavior in detail, we investigated microstructural aspects of food intake in a controlled laboratory experiment. Eating behavior of 43 obese women and 42 normal weight controls between 18 and 30 years was assessed twice (one time after participating in the Trier Social Stress Test and one time after a control session). A universal eating monitor was used to obtain cumulative intake curves with pudding as laboratory food. Compared to controls, obese showed inhibited eating behavior after stress. The results are interpreted and discussed with regard to restrained eating.
\end{abstract}

Keywords: Eating Behavior; Laboratory Food Intake; Stress; Obesity; Restrained Eating

\section{Introduction}

The identification of factors which can be causally linked to overeating is important to clarify the etiology of obesity. According to Schachter's 'externality theory of obesity' [1], eating behavior of obese people is more reactive to external cues (time, presence of food, situational effects) and less sensible to internal hunger and satiety signals than in normal weight people. But the relationship between high external responsiveness, overeating and weight gain is complex. Emotions and cognitions are also important factors which influence eating behavior [2-5]. Negative emotions and stress as well as increased cognitive concerns about food intake and weight regulation may disturb mechanisms of a balanced eating behavior by inhibition or stimulation of food intake, independent of hunger or satiety. Both play an important role to promote obesity [6].

To investigate eating behavior in detail one has to look at microstructural aspects of food ingestion [7] that represent intake characteristics during a laboratory meal by measuring cumulative intake of the test meal. Background is a two process theory of ingestion that separates between the beginnings of a meal, subjectively perceived as hunger and the end of a meal subjectively perceived as satiety. The measurement tool is a universal eating monitor (UEM) [8] and is of proven reliability [9]. The UEM generates the following characteristics of the eating style during the laboratory meal: initial eating rate (IER), average eating rate (AER), change of eating rate (CER) in the sense of acceleration or deceleration, size of spoonfuls (SSF), and total intake (TI).

Studies comparing eating behavior in laboratory obtaining cumulative intake curves by UEM showed differences in eating style between obese and normal weight subjects [10-12]. Obese people showed higher average eating rates [13-15], higher initial eating rates, a larger size of spoonfuls and greater food intake [12] compared to normalweight subjects. However, not all studies found differences in eating style [11,14]. Adams et al. [15] reported neither a greater amount of consumed food nor a higher number of bites or rates of chewing in obese. Spiegel [16] also found no significant differences between obese and non-obese subjects.

Stress has been hypothesized to lead to high short-term caloric intake which results in weight gain in the long-term. Empirical data, however, are inconsistent $[2,17]$. This might be mainly due to looking only at total intake and not to the microstructural aspects of eating.

As an important moderating factor for Disinhibition of eating behavior after stress restrained eating has been proposed. Dietary restraint describes the intention of individuals to restrict their food intake in order to achieve weight loss or to prevent weight gain. Restrained eaters cognitively instead of physiologically control their eating behavior under normal circumstances. The behavioral strategies involve for example deliberately limiting one's food intake per day, calculating consumed energy, avoiding high calorie 
dishes, ending a meal before reaching satiety, and frequent weighing. Usually, obese people score high on questionnaires that measure restrained eating [18].

To understand how stress affects eating behavior of obese and normal weight women the present study investigated the effect of an acute psychosocial stressor on the microstructure of eating behavior in obese and normalweight women. Furthermore, we analyzed the influence of restrained eating.

\section{Method}

Eighty-five healthy women aged 18 to 30 years were recruited through advertisement at the University of Trier, Germany. 43 were obese (BMI $31.5 \mathrm{~kg} / \mathrm{m}^{2} \pm 1.8$ ) and 42 were normal weight (BMI $\left.21.7 \mathrm{~kg} / \mathrm{m}^{2} \pm 2.0\right)$. Exclusion criteria were smoking, regular alcohol consumption (more than seven drinks a week), drug or medicine use, because these factors may affect cortisol reactivity [19,20]. To assess the presence of any of these exclusion criteria participants were examined and interviewed by a physician, who also asked for the present stage of the menstrual cycle of the participants. No systematic differences in cycle phase were detected between obese subjects and the control group.

All women received $€ 80$ for their participation. The study was approved by the ethics committee of the University of Trier in April 2012. All subjects gave oral and written informed consent.

\section{Procedure}

Participants were exposed to two days of two-hour laboratory sessions, starting at $2.00 \mathrm{pm}$ each day. The maximum time period between the two laboratory sessions was one week. The process of every session was equal except for the condition (stress vs. neutral). In the stress condition participants took part in the Trier Social Stress Test [21], a standardized laboratory stressor designed to elicit psychobiological stress responses. The TSST consisted of a three-minute speech preparation period, a five-minute public speaking task in front of two evaluative, non-responsive audience members and a five-minute mental arithmetic task. In the neutral condition participants read magazines for the same period of time. The sequence of neutral and stress condition was randomized. To create standardized internal state of satiety, participants were asked to refrain from eating and drinking (excluding water) for three hours before each session. On arrival at the laboratory all subjects were asked to confirm that they had followed the instructions.

Salivary cortisol samples were collected at the same time intervals throughout each session at four measurement points: baseline, + $20 \mathrm{~min},+30 \mathrm{~min},+60 \mathrm{~min}$ after the TSST or control session. In both sessions (stress and neutral condition) participants made subjective ratings of their current stress level on $100 \mathrm{~mm}$ visual analogue scales (VAS; 0: not at all, 100: maximum). After the TSST or control session, participants had a test meal at the universal eating monitor (UEM) [22]. As test meal participants were served $500 \mathrm{~g}$ of chocolate pudding. Nutritional content per $100 \mathrm{~g}$ was: $158 \mathrm{kcal}, 3,1 \mathrm{~g}$ protein, $15,8 \mathrm{~g}$ carbohydrate, $9,1 \mathrm{~g}$ fat). They were instructed to eat as much of the meal as they liked. Before subjects ate the test meal they rated their hunger feeling by visual analogue scales (VAS; 0: not at all, 100: maximum) Ratings of subjective stress before and after the TSST were also obtained by such scales.

\section{Biochemical analysis of salivary cortisol}

Saliva samples were collected using salivettes (Sarstedt, Nümbrecht, Germany), plastic vials with cotton dental rolls inside, and frozen at $-20{ }^{\circ} \mathrm{C}$ until laboratory analysis. They were assayed using delayed Fluoreszenz-Immunoasseys. Intraassay coefficients of variation were $4.0-6.7 \%$. The interassay coefficient of variation was $7.1-9.0 \%$ [23].

All samples were tested in duplicate. Values used in data analyses are the averages of duplicate tests. Cortisol data were converted to $\mathrm{nmol} / \mathrm{l}$.

\section{Measurement of eating behavior}

The following characteristics of the eating style during the laboratory meal were measured by a universal eating monitor: initial eating rate (IER), average eating rate (AER), change of eating rate (CER) in the sense of acceleration or deceleration, size of spoonfuls (SSF), and total intake (TI).

The degree of dietary restraint was assessed by the German version of the Three Factor Eating Questionnaire (factor "cognitive control of eating") [18].

\section{Statistical analysis}

Salivary cortisol and subjective stress ratings before and after the TSST were analyzed by 2 factorial ANOVA with between subjects factor "group" (obese vs normalweight) and within subjects factor "stress" (rating before and after TSST; cortisol increase after TSST).

To analyze the microstructure of eating behavior 2-factorial ANCOVA for repeated measurement was used. Between subjects 
factor was group (obese vs. normalweight), within subjects factor was condition (stress vs. neutral). Dependent variables were average eating rate (AER), initial eating rate (IER), change of eating rate (CER), size of spoonfuls (SSF) and total intake (TI). Restrained eating was included as covariate. Significance levels are all 2-tailed. Partial ETA squared served as a measure of effect size. All statistical analyses were performed using SPSS version 21.

\section{Results}

\section{Manipulation check of stress}

The rise in subjective stress after the TSST was significant for both comparison groups $\left(\mathrm{F}(2,148)=24,29, \mathrm{p}<.001, \eta^{2}=.25\right)$. There was also a significant stress-induced increase in salivary cortisol over all subjects $\left(\mathrm{F}(3,240)=31,88, \mathrm{p}<.001, \eta^{2}=.29\right)$.

\section{Eating behavior}

Table 1 shows the intake characteristics for the laboratory meal for the obese and normal weight subjects in the stress and neutral condition.

\begin{tabular}{|c|c|c|c|c|}
\hline & Obese (stress) & Obese (neutral) & $\begin{array}{c}\text { Normalweight } \\
\text { (stress) }\end{array}$ & $\begin{array}{c}\text { Normalweight } \\
\text { (neutral) }\end{array}$ \\
\hline AER $(\mathrm{g} / \mathbf{s})$ & $.80 \pm .36$ & $.89 \pm .39$ & $.96 \pm .43$ & $.89 \pm .38$ \\
\hline IER $(\mathrm{g} / \mathbf{s})$ & $1.11 \pm .64$ & $1.24 \pm .76$ & $1.19 \pm .62$ & $1.06 \pm .51$ \\
\hline CER $\left(\mathbf{g} / \mathbf{s}^{2}\right)$ & $1.25 \pm 1.37$ & $1.32 \pm 1.82$ & $1.13 \pm 1.07$ & $.81 \pm 1.06$ \\
\hline TI $(\mathrm{g})$ & $233.40 \pm 99.42$ & $293.36 \pm 101.61$ & $256.29 \pm 133.52$ & $275.77 \pm 125.14$ \\
\hline SSF $(\mathrm{g})$ & $12.24 \pm 3.91$ & $12.57 \pm 4.02$ & $12.48 \pm 4.67$ & $12.22 \pm 4.38$ \\
\hline
\end{tabular}

AER: Average Eating Rate; IER: Initial Eating Rate; CER: Change of Eating Rate); TI: Total Intake; SSF: Size of Spoonfuls

Table 1: UEM-data of obese and normal weight women $(\mathrm{M} \pm \mathrm{SD})$

The interaction between group and condition was significant for $\operatorname{AER}\left(\mathrm{F}(1,64)=2.94 ; \mathrm{p}<.05 ; \eta^{2}=.04\right)$. Obese subjects ate slower after stress than the normal weight women, whereas the obese women ate faster than the normal weights in the neutral condition. Restrained eating had an influence on $\operatorname{AER}\left(\mathrm{F}(1,64)=5.27, \mathrm{p}<.02, \eta^{2}=.08\right)$. High scores on restrained eating resulted in slower eating $(\mathrm{r}=-.37, \mathrm{p}<.01)$.

For IER there was an interaction of group $\mathrm{x}$ condition $\left(\mathrm{F}(1,64)=4.20, \mathrm{p}<.04 ; \eta^{2}=.06\right)$. After stress obese subjects had a lower IER than in the control session, whereas normal weight women had a higher IER after stress than in the control session. Restrained eating had a significant influence on $\operatorname{IER}\left(\mathrm{F}(1,64)=9.69, \mathrm{p}<.001, \eta^{2}=.13\right)$. The higher a subject scored on restrained eating, the slower she ate at the beginning of the test meal $(\mathrm{r}=-.34, \mathrm{p}<.02)$.

No significant group differences in CER after stress were found, but restrained eating had a significant effect on CER $(\mathrm{F}(1,60)=$ 5.07; $\left.\mathrm{p}<.02, \eta^{2}=.08\right)$. Higher scores in restrained eating were associated with more acceleration of eating rate towards the end of the laboratory meal $(\mathrm{r}=.37, \mathrm{p}<.01)$.

No significant group differences in SSF after stress could be shown, but restrained eating had a significant effect on SSF $(\mathrm{F}(1,63)=$ $\left.5.76, \mathrm{p}<.01, \eta^{2}=.08\right)$. High scores on restrained eating resulted in smaller SSF. $(\mathrm{r}=-.33, \mathrm{p}<.02)$.

For TI, the effect of condition was significant $\left(\mathrm{F}(1,66)=11,47, \mathrm{p}<.001 ; \eta^{2}=.15\right)$. In the control session subjects ate more than in the stress condition. No significant group differences were detected and there was no significant influence of restrained eating on total intake.

\section{Discussion}

In the present study we investigated whether acute psychosocial stress influences microstructure of eating behavior in obese and normal weight women. Furthermore, we analyzed the role of restrained eating.

In our study the amount of food intake did not differ between obese and normal weight women. This result confirms studies which also found no weight related differences [24]. Moreover, we found a stress-induced inhibition of food intake in all subjects. In the control condition all subjects ate more of the test meal than in the stress condition. Our results are consistent with previous studies proposing that stress inhibits food intake [25]. But the analysis of microstructural characteristics of eating behavior showed that stress inhibited eating behavior especially in obese subjects whereas a stress-induced stimulation was detected in normal weight subjects. With regard to initial eating rate, obese subjects ate slower at the beginning of the meal after stress compared to the control condition. The normal weight subjects ate faster after the exposure to stress than after the neutral task. Initial eating rate in the laboratory meal can be interpreted as a behavioral indicator for hunger [7]. Spiegel [16] suggests that obese subjects are less hungry than lean subjects. Our results confirm this assumption only in part. After stress, obese participants had slower initial eating rates, so hunger seemed to be inhibited. In the neutral condition, however, obese subjects showed higher hunger through 
faster initial eating rate. The results of total intake are in line with this interpretation. In the neutral condition, obese people ate more than after stress. On the other hand, in the neutral condition obese subjects showed an adverse eating style through faster initial and average eating rates. This can promote overconsumption in a single meal, because fast eating may disturb perception of satiety.

High values on restrained eating did not lead to greater food intake but influenced microstructural characteristics of eating behavior. Restrained eating was mirrored in an inhibited eating style. High values on restrained eating came along with a smaller size of spoonfuls, a slower initial eating rate, a lower average eating rate and more acceleration of eating rate towards the end of the meal. Zandian et al. [26] and Westerterp-Plantenga [14] found a constant eating rate in restrained eaters. This eating style points to a significant deficit in the adequate perception of hunger and satiety in obese people that is responsible for the maintenance of the overweight in the long-term.

There are several mechanisms that have been proposed to be involved in the regulation of eating behavior after stress. The models of stress and eating that have been empirically tested fall into two basic categories. The first type predicts that stress will increase eating in all exposed organisms. This model has been tested primarily in animals. Positing a general response, this model lends itself to physiological explanations of stress-induced eating, and most research testing the general model has been directed toward finding physiological pathways to explain stress-induced eating.

The second type of model focuses on individual differences. This model that has been tested in humans, posits that individual differences in learning history, attitudes, or biology determine the effects of stress on eating. Models of this type predict that identifiable groups will differ in their eating when they are stressed. Three ways of identifying individuals who will be predisposed to stress-induced eating have been proposed. One proposal is that normal-weight individuals decrease their eating while stressed, whereas eating by obese individuals is unaffected by stress. Another proposal is that people who must work to control their eating (i.e., "restrained" individuals) increase their eating while stressed, whereas people who do not have to work to control their eating are unaffected by stress. Finally, it has been proposed that women are more likely to eat under stress than men, particularly certain food.

This study has limitations that should be taken into account when the results are interpreted. The sample size was small. A replication with a larger sample is required. Only women were included in the sample because eating disorders and obesity are more common in females than in males [27]. The study was conducted in a laboratory setting, which has the advantage to control interfering variables, but the disadvantage of a limited generalization to the natural environment. But there is empirical evidence, that such laboratory results can be transferred to the eating behavior of clinical eating disorders in their daily environment [28].

The present results imply that treatment programs for obesity should improve the perception of internal cues for hunger and satiety.

\section{Conclusion}

The presented results provide evidence that obese women show inhibition of microstructural aspects of eating behavior during a laboratory meal after stress. The determining factor was the degree of dietary restraint that was higher in obese compared to normal weight controls. The study underscores the significance of laboratory stress tests in obesity in particular with regard to treatment recommendations and prevention of the maintenance of the disease.

\section{References}

1. Schachter S (1971) Some extraordinary facts about obese humans and rats. Am Psychol 26: 129-44.

2. Greeno CG, Wing RR (1994) Stress-induced eating. Psychol Bull 115: 444-64.

3. Ganley RM (1989) Emotion and eating in obesity: A review of the literature. Int J Eat Disorder 8: 343-61.

4. Macht M, Simons G (2000) Emotions and eating in everyday life. Appetite 35: 35-71.

5. Slochower J (1976) Emotional labeling and overeating in obese and normal weight individuals. Psychosom Med 38: 131-9.

6. Yau YHC, Potenza MN (2013) Stress and eating behaviors. Minerva Endocrinol 38: 255-67.

7. Guss J, Kissileff H (2000) Microstructural analyses of human ingestive patterns: from description to mechanistic hypotheses. Neurosci Biobehav Rev 24: 261-8.

8. Kissileff HR, Thornton J, Becker E (1982) A quadratic equation adequately describes the cumulative food intake curve in man. Appetite 3: 255-72.

9. Hubel R, Laessle RG, Lehrke S, Jass J (2006) Laboratory measurement of cumulative food intake in humans: results on reliability. Appetite 46: 57-62.

10. Spitzer L, Rodin J (1981) Human eating behavior: a critical review of studies in normal weight and overweight individuals. Appetite 2: $293-329$.

11. Rodin J, Mancuso J, Granger J, Nelbach E (1991) Food cravings in relation to body mass index, restraint and estradiol levels: a repeated measures study in healthy women. Appetite 17: 177-85.

12. Laessle RG, Lehrke S, Dückers S (2007) Laboratory eating behavior in obesity. Appetite 49: 399-404.

13. Barkeling B, Linné Y, Melin E, Rooth P (2003) Vision and eating behavior in obese subjects. Obes Res 11: 130-4.

14. Westerterp-Plantenga MS, Wouters L, ten Hoor F (1991) Restrained eating, obesity, and cumulative food intake curves during four-course meals. Appetite 16: 149-58.

15. Adams N, Ferguson J, Stunkard AJ, Agras S (1978) The eating behavior of obese and nonobese women. Behav Res Ther 16: 225-32. 
16. Spiegel TA (2000) Rate of intake, bites, and chews - the interpretation of lean-obese differences. Neurosci Biobehav Rev 24: 229-34.

17. Oliver G, Wardle J (1999) Perceived effects of stress on food choice. Physiol Behav 66: 511-5.

18. Pudel VV, Westenhöfer J (1989) Der Fragebogen zum Eßverhalten (FEV) Hogrefe, Göttingen, Germany.

19. Kirschbaum C, Hellhammer DH (1989) Salivary cortisol in psychobiological research: an overview. Neuropsychobiology 22: 150-69.

20. Pruessner J, Gaab J, Hellhammer D, Lintz D, Schommer N, et al. (1997) Increasing correlations between personality traits and cortisol stress responses obtained by data aggregation. Psychoneuroendocrinology 22: 615-25.

21. Kirschbaum C, Pirke KM, Hellhammer DH (1993) The 'Trier Social Stress Test'-a tool for investigating psychobiological stress responses in a laboratory setting. Neuropsychobiology 28: 76-81.

22. Kissileff HR, Klingsberg G, Van Itallie TB (1980) Universal eating monitor for continuous recording of solid or liquid consumption in man. Am J Physiol 238: R14-22.

23. Dressendörfer RA, Kirschbaum C, Rohde W, Stahl F, Strasburger CJ (1992) Synthesis of a cortisol-biotin conjugate and evaluation as a tracer in an immunoassay for salivary cortisol measurement. J Steroid Biochem 43: 682-93.

24. Spiegel TA, Kaplan JM, Tomassini A, Stellar E (1993) Bite size, ingestion rate, and meal size in lean and obese women. Appetite 21: 131-45.

25. Torres SJ, Nowson CA (2007) Relationship between stress, eating behavior, and obesity. Nutrition 23: 887-94.

26. Zandian M, Ioakimidis I, Bergh C, Brodin U, Södersten P (2009) Decelerated and linear eater: Effect of eating rate on food intake and satiety. Physiol Behav 96: $270-5$.

27. Flegal KM, Carroll MD, Kuczmarski RJ, Johnson CL (1998) Overweight and obesity in the United States: prevalence and trends, 1960-1994. Int J Obes Relat Metab Disord 22: 39-47.

28. Kissileff HR, Walsh BT, Kral JG, Cassidy SM (1986) Laboratory studies of eating behavior in women with bulimia. Physiol Behav 38: 563-70.

29. Laessle R, Geiermann L (2012) Reliability of laboratory measurement of human food intake. Appetite 58: 249-51.

30. Tanofsky-Kraff M, Wilfley De, Spurrell E (2000) Impact of interpersonal and ego-related stress on restrained eaters. Int J Eat Disord 27: 411-8.

31. Wallis DJ, Hetherington MM (2004) Stress and eating: the effects of ego-threat and cognitive demand on food intake in restrained and emotional eaters. Appetite 43: 39-46. 